\title{
Overweight, obesity and breast cancer prognosis: optimal body size indicator cut-points
}

\author{
Bilal Majed · Thierry Moreau · Bernard Asselain • \\ Curie Institute Breast Cancer Group
}

Received: 13 May 2008/Accepted: 14 May 2008/Published online: 12 June 2008

(C) Springer Science+Business Media, LLC. 2008

\begin{abstract}
Background Evidence from the data provided in numerous published articles indicates that obesity and overweight can have a negative prognosis role in breast cancer. However, different Body Size Indicators (BSI) and cut-points have been employed and may partly explain discrepancies between the findings of various studies. Material and methods 14,709 women were recruited, treated and followed for a first unilateral breast cancer. After randomly splitting the patients' data into two groups, a maximum statistical outcome approach was used to select optimal BSI cut-points from a "training sample", when prognosis events were investigated. External validation was then carried out using a "validation sample", and agreement between the selected optimal BSI cut-points was assessed. Body Mass Index (BMI), weight (W), Ideal Weight Ratio (IWR) and Body Surface Area (BSA) were used, and were assessed at the time of diagnosis. Results The selected optimal BSI cut-points were reliable when overall survival, metastasis recurrence and disease free
\end{abstract}

B. Majed · B. Asselain

Biostatistics Department, Curie Institute (cancer research

and treatment institute), 26 rue d'Ulm, 75005 Paris, France

B. Majed ( $\square)$

12 rue Dambrine, 62000 Arras, France

e-mail: bmajed@free.fr

T. Moreau

INSERM UNIT 780: Epidemiology and biostatistics,

Paul-Brousse Hospital, 16 avenue Paul Vaillant Couturier,

94807 Villejuif Cedex, France

e-mail: moreau@vjf.inserm.fr

B. Asselain - Curie Institute Breast Cancer Group

Paris, France

e-mail: bernard.asselain@curie.net interval events were investigated. The chosen BMI cutpoint values matched the overweight cut-point value given by the World Health Organization. Agreement between defined binary BSI was acceptable; however, it varied from "fair" to "very good". Analysis of second primary cancer occurrence and contralateral recurrence events was not conclusive. When local and node recurrence events were taken into account, the results were inconsistent and were linked to an unconfirmed relationship between stoutness and these prognosis events. Conclusions Efficient, optimal BSI cut-points indicate a poorer prognosis, illustrated by a shortened overall survival and an increase of metastasis recurrences, from a BMI value of $25 \mathrm{~kg} / \mathrm{m}^{2}$, a W value of $60 \mathrm{~kg}$, an IWR value of $20 \%$ and a BSA value of $1.7 \mathrm{~m}^{2}$. Further BSI cut-point investigations are needed, taking into account contralateral recurrence and second primary cancer events.

Keywords Breast cancer - Prognosis . Body size indicators $\cdot$ Cut-points $\cdot$ External validation

\section{Introduction}

Numerous relevant studies support the negative prognosis role of obesity and/or overweight in female breast cancer [1-5]. Nonetheless, significant discrepancies between methodologies and results have been observed, and some findings support the controversy surrounding this topic. One of the limitations of this type of assessment is the use of various stoutness indicators and cut-points in order to define overweight and obesity [1].

The Body Mass Index (BMI) is often used according to the recommendations of the World Health Organization (WHO) [6]. Although the WHO has defined recommended 
BMI cut-points $\left(18.5 ; 25\right.$ and $\left.30 \mathrm{~kg} / \mathrm{m}^{2}\right)$, other values are used in the literature dealing with breast cancer prognosis: the cut-points range extends from 21 to $30 \mathrm{~kg} / \mathrm{m}^{2}$ in studies exploring the influence of excess of weight in the overall survival (the most frequently investigated prognosis event) of women with breast cancer [1]. In these surveys, significant estimated effects vary largely according to the choice of cut-points. Though BMI is known to provide a reliable assessment of the prevalence of obesity and overweight on the scale of a population [6-8], it also suffers from some limitations [9]. The same cut-off value may correspond to different degrees of fatness, depending on the studied populations [6, 10]. Moreover, Stevens et al. [11] observed that "BMI cut-offs associated with equivalent risk across ethnic groups differ widely depending on the outcome and the risk estimate".

Other Body Size Indicators (BSI), obtained from weight (W) and height $(\mathrm{H})$, have also been considered in breast cancer prognosis, including weight itself [12-19], Ideal Weight Ratio (IWR) [18, 20-22] and Body Surface Area (BSA) [12, 18]. Kyogoku et al. [18] showed that there are strong quantitative correlations between BSI themselves, whilst significant multivariate breast cancer prognosis effects were observed when BSI were coded as categorical variables. However, Himes et al. [23] observed a lack of correspondence between some measures, commonly used to identify obesity, when the BSI cut-points were defined using the distributions' fifth quintile. This study revealed a potential classification bias, linked to the choice of BSI cutpoints used to define overweight or obesity, whereas strong quantitative correlations were observed between stoutness indicators computed from height and weight.

As a result of the multiplicity of published overweight and obesity definitions [1], whose influence is taken into account in breast cancer prognosis, the reliability of BSI and cut-point choices can be questioned. Furthermore, as excess of weight can have potentially clinical implications linked to nutritional habits [24], BSI (which can be easily obtained from weight and height) should be preferred to other invasive and costly fatness evaluation tools for the purposes of breast cancer prognosis. In this context, the continuous use of stoutness indicators would not be useful, whereas the use of binary coding to oppose heavier patients to others would indeed be more relevant. In a previous paper [5], the authors of the present paper confirmed, using recommended WHO BMI cut-points, the independent role of obesity in the prognosis of female breast cancer. Multivariate analyses revealed relevant overweight and obesity effects related to metastasis recurrences, overall survival and second primary cancer occurrences. Knowing that the choice of BSI cut-points can have an influence on the association between the stoutness and the prognosis of the breast cancer, the purpose of this study was to establish and validate the most appropriate and informative cutpoints for different BSI. In addition, the agreement between selected optimal BSI cut-points was evaluated.

\section{Material and methods}

\section{Material}

The characteristics of our population are summarized in a previously published paper [5]. We selected 14,709 women who were recruited, treated and followed at the Curie Institute (Paris-France) for a first unilateral breast cancer without distant dissemination. The patients were recruited between 1981 and 1999 and were followed prospectively. Less than $15 \%$ of the initially eligible patients were excluded because of missing anthropometric data. Height and weight were measured at the time of diagnosis.

Methods

Previous published analyses [5] of this population revealed significant obesity prognosis effects using a categorical BMI variable to define overweight and obesity, according to WHO recommendations. In this study, our aim was to define the most suitable BSI cut-points, in terms of their relevance to several prognosis events in breast cancer: local, nodal, contralateral and distant recurrences, overall survival and second primary cancer occurrences. The Disease Free Interval (DFI), including the absence of metastases, nodal or local recurrences, was also explored. We thus computed several BSI from the patients' weight and height: BMI expressed in $\mathrm{kg} / \mathrm{m}^{2}, \mathrm{~W}$ in $\mathrm{kg}$, IWR $\left[\left(1-\frac{W}{(H-100) * 0.9}\right) * 100\right]$ in $\%$ and BSA according to the formula given by Gehan and George: $\left[0.0235 * H^{0.42246} * W^{0.51456}\right]$ in $\mathrm{m}^{2}(\mathrm{H}$ is in centimeters and $\mathrm{W}$ is in kilograms) $[18,25]$.

The search for optimal binary BSI cut-offs was supported by an observed continuous monotonous relationship between BSI and prognosis events in the studied population (data not shown). The range of explored cut-offs for each BSI was based on the observed descriptive statistics of these indicators in the studied population, and the published BSI cut-points employed in breast cancer prognosis studies (Table 1). The optimal BSI cut-points were selected by using a maximum statistical outcome method. Its goal was to select BSI values that best separated low and high risk patients, according to the studied outcome. We used Cox proportional hazards models [26] based on a partial likelihood maximization, for the estimation of parameters, in order to appreciate the univariate prognosis effect of BSI cut-points. We employed the Log Likelihood (LL) and the 
Table 1 BSI descriptive statistics and cut-off range explored in our study

\begin{tabular}{lccccc}
\hline BSI & Mean \& 95\% CI & Quintile 25\% & Quintile 50\% & Quintile 75\% & $\begin{array}{c}\text { Explored BSI } \\
\text { cut-points range }\end{array}$ \\
\hline BMI $\left(\mathrm{kg} / \mathrm{m}^{2}\right)$ & $23.76[23.69-23.82]$ & 20.94 & 23.05 & 25.72 & $18-40$ \\
IWR $(\%)$ & $12.44[12.12-12.76]$ & -1.43 & 9.26 & 22.22 & $5-55$ \\
W $(\mathrm{kg})$ & $61.96[61.78-62.14]$ & 54 & 60 & 68 & $50-85$ \\
BSA $\left(\mathrm{m}^{2}\right)$ & $1.68[1.67-1.68]$ & 1.56 & 1.66 & 1.77 & $1.25-2.25$ \\
\hline
\end{tabular}

Akaike Information Criterion (AIC) of the Cox models, which are known to be reliable tools for the selection of variables in a modeling process [27-29]. They enable, respectively, nested and non-nested models to be compared. Harrell's C-index [28, 30], a tool used for the assessment of model discrimination ability, was also used. It represents the probability of concurrence between model predicted values and accurate outcomes. In survival data, it is computed by using all informative pairs of subjects (at least one subject in each given pair is not censored), for which predicted and observed survival times are compared. As presented by Pencina and D'Agostino [31], the overall $\mathrm{C}$ index is "the proportion of usable pairs in which the predictions and outcomes are concordant". A C-index value of 0.5 is considered to result from random predictions, whereas a value of 1 corresponds to perfectly accurate predictions [28]; however, poor C-index values can be induced by a high number of censored times (Table 2). After having selected the optimal BSI cut-points for a given prognosis event, we assessed the agreement between the cut-points using the kappa coefficient [32]. Continuous correlations between the BSI themselves were assessed using a correlation coefficient [33].

The definition of 'best' BSI thresholds according to statistical criteria, has been associated with an inflation of type I error rates, and a tendency towards the overestimation of measured effects [34]. Therefore, by taking advantage of data based on a large population and a considerable number of prognosis events (Table 2), we achieved an external validation strategy [28] based on data splitting: we randomly selected $70 \%$ of our initial population data, thereby defining a "training or test sample"
( $N=10,296)$, to perform "optimal" BSI cut-points choices for each prognosis event. The results obtained were then applied to the other patients, i.e. the remaining $30 \%$ of our initial population, thereby defining a "validation sample" $(N=4413)$. Whereas univariate results were used to select "optimal" BSI thresholds, multivariate adjustments were then computed using the principal breast cancer prognosis factors: age, menopausal status, size of tumor, tumor's Scarf-Bloom-Richardson grade, year of diagnosis (recruitment), expression of estrogen and progesterone receptors by the tumor, local and lymph node invasion, number of invaded nodes after axillary dissection, type of surgical treatment, use of hormonotherapy, chemotherapy and/or radiotherapy. The obesity prognosis effect is known to be linked to breast cancer characteristics at the time of diagnosis [5, 35], as it may be linked to a delayed diagnosis. Multivariate adjustments were therefore computed. As common breast cancer prognosis factors are not useful for the assessment of the risk of second primary cancers, only age and menopausal status were used as multivariate adjustments for the study of second primary cancer events. Hazard Ratios (HR), corresponding to each binary BSI effect, were computed with $95 \%$ confidence intervals (95 CI) and LL ratio tests [27, 28].

Power tests linked to the adjusted HR corresponding to the validation sample, which represented $30 \%$ of the initial population, were estimated in order to improve the interpretation of results. A substantial loss of power was expected. The Freedman formula, used in Cox proportional models, proposed by Rosner [33], was used to estimate power test outcomes. The S-plus statistical software was used for data analysis.
Table 2 Number of events and proportion of uncensored survival times

\begin{tabular}{lccc}
\hline Events & $\begin{array}{l}\text { Global Population } \\
(N=14709)\end{array}$ & $\begin{array}{l}\text { Training Sample } \\
(N=10296)\end{array}$ & $\begin{array}{l}\text { Validation Sample } \\
(N=4413)\end{array}$ \\
\hline Overall survival & $3693(25.1 \%)$ & $2558(24.8 \%)$ & $1135(25.7 \%)$ \\
Metastasis recurrence & $3780(25.7 \%)$ & $2634(25.6 \%)$ & $1146(26 \%)$ \\
Local recurrence & $2069(14.1 \%)$ & $1442(14 \%)$ & $627(14.2 \%)$ \\
Nodal recurrence & $323(2.2 \%)$ & $227(2.2 \%)$ & $96(2.2 \%)$ \\
Contralateral recurrence & $1009(6.9 \%)$ & $690(6.7 \%)$ & $319(7.2 \%)$ \\
Disease free interval & $4876(33.1 \%)$ & $3416(33.2 \%)$ & $1460(33.1 \%)$ \\
Second cancer & $555(3.8 \%)$ & $380(3.7 \%)$ & $175(4 \%)$ \\
\hline
\end{tabular}




\section{Results}

High significant correlations were observed between BSI indicators when they were used as continuous variables. The highest correlations were found between BMI and IWR (the correlation coefficient reached the value of 0.99 ) and between Weight and BSA (0.98); the lowest correlation coefficient was observed between IWR and BSA (0.74).

From the training sample, the agreement between LL, AIC and C-index best values (obtained from the univariate analysis), enabled optimal BSI cut-points to be selected when all prognosis events were considered. The results in terms of overall survival, metastasis recurrence and DFI events indicated that unadjusted and adjusted HR estimates from the training sample were reproducible in the validation sample (Table 3a). Nonetheless, in the validation sample, adjusted HR were often statistically non-significant and estimated power tests were frequently poor (data not shown). DFI evaluations made with the validation sample presented comparatively less significant results. The Kappa agreement quality [36] between selected BSI cut-offs varied from "fair" to "very good" (Table 4). The best kappa coefficients were observed between BMI and IWR (0.90), and between $\mathrm{W}$ and BSA (0.84), when overall survival outcome was evaluated. Optimal BMI cut-points, linked to overall survival, metastasis recurrences and DFI, corresponded to the overweight threshold value according to the WHO definition. Concerning second primary cancer and contralateral recurrence outcomes, significant univariate and multivariate effects were observed in the training sample (Table 3b). However, in the case of contralateral recurrences, with the exception of BSA, reduced HR estimations were found in the validation sample. This phenomenon was more pronounced for second primary cancer events, for which the estimated HR were half of those from the training sample. Once again, quality agreements between defined best binary BSI for these outcomes varied from "fair" to "very good". The best agreements were observed between BMI and IWR (0.92), and between $\mathrm{W}$ and BSA (0.90), when contralateral recurrence outcomes were investigated; the BMI optimal cut-point matched the threshold value of overweight defined by the WHO. Concerning the analysis of second primary cancer outcomes, the optimal BMI cut-point was $29 \mathrm{~kg} / \mathrm{m}^{2}$ which closely matched the WHO threshold value for obesity. In this case, the BMI cut-off had a modest ("fair") kappa coefficient when compared with IWR, whereas $\mathrm{W}$ agreed much better with the BSA.

The study of local recurrence events led, in most cases, to non-significant HR from both training and validation samples (Table 3c). HR estimations were often close to the unity, except for the case of BSA: the choice of a $2.2 \mathrm{~m}^{2}$ BSA cut-off led to $0.6 \%$ of the test sample patients being defined as "stout", and to fewer uncensored times, whereas other BSI cut-points led to a far greater number of patients being defined as overweight. When node recurrence events were taken into account, few statistically significant results were observed from the training sample (univariate and multivariate tests of $\mathrm{W}$ and BSA). HR values estimated from the validation sample were contradictory to those determined using the training sample. Very poor kappa coefficients were found between some optimal binary BSI, when local and nodal recurrences were explored (considering, respectively, BSA and IWR).

Univariate associations between optimal binary BSI and prognostic events were frequently linked to poor $\mathrm{C}$-index values (close to 0.5 ). Multivariate adjustments improved the C-index values to nearly $0.70-0.75$ in almost all cases, except for those characterized by second cancer and contralateral events for which multivariate adjustments very slightly improved this index (Table 5). In the case of second cancer events, C-index values from the training sample were clearly higher than those from the validation sample. Concerning node recurrence events, $\mathrm{C}$-indices values linked to adjusted HR values from the validation sample were clearly higher than those derived from the training sample.

Finally, optimal BMI cut-points were frequently characterized by the best LL, AIC and C-index values, linked to breast cancer prognosis analyses, even though, the other BSI had values very close to those given by the BMI.

\section{Discussion}

This study, carried out using a methodology which implements an external validation, based on data splitting, has enabled optimal BSI cut-points to be identified and associated to the prognosis of breast cancer. When compared with other published studies which investigate the prognostic role of obesity and/or overweight [1] in breast cancer, the significant number of events and large population size, reported here, ensure that HR estimations were derived with a reliable effect size beyond the statistical tests significance. Nevertheless, in a simulation study, Faraggi and Simon [34] found that a maximum statistically selected variable cut-point can lead to an overestimated HR. This phenomenon would be related to the HR achieved from our training sample.

Selected optimal BSI cut-points were effective in terms of breast cancer prognosis when overall survival (OS), metastasis recurrence and DFI events were studied. As the BMI selected threshold values were 24 and $25 \mathrm{~kg} / \mathrm{m}^{2}$, the role of excess weight in breast cancer prognosis starts to become significant at the WHO-defined threshold (cutpoint) for overweight. The W, IWR and BSA cut-offs are 


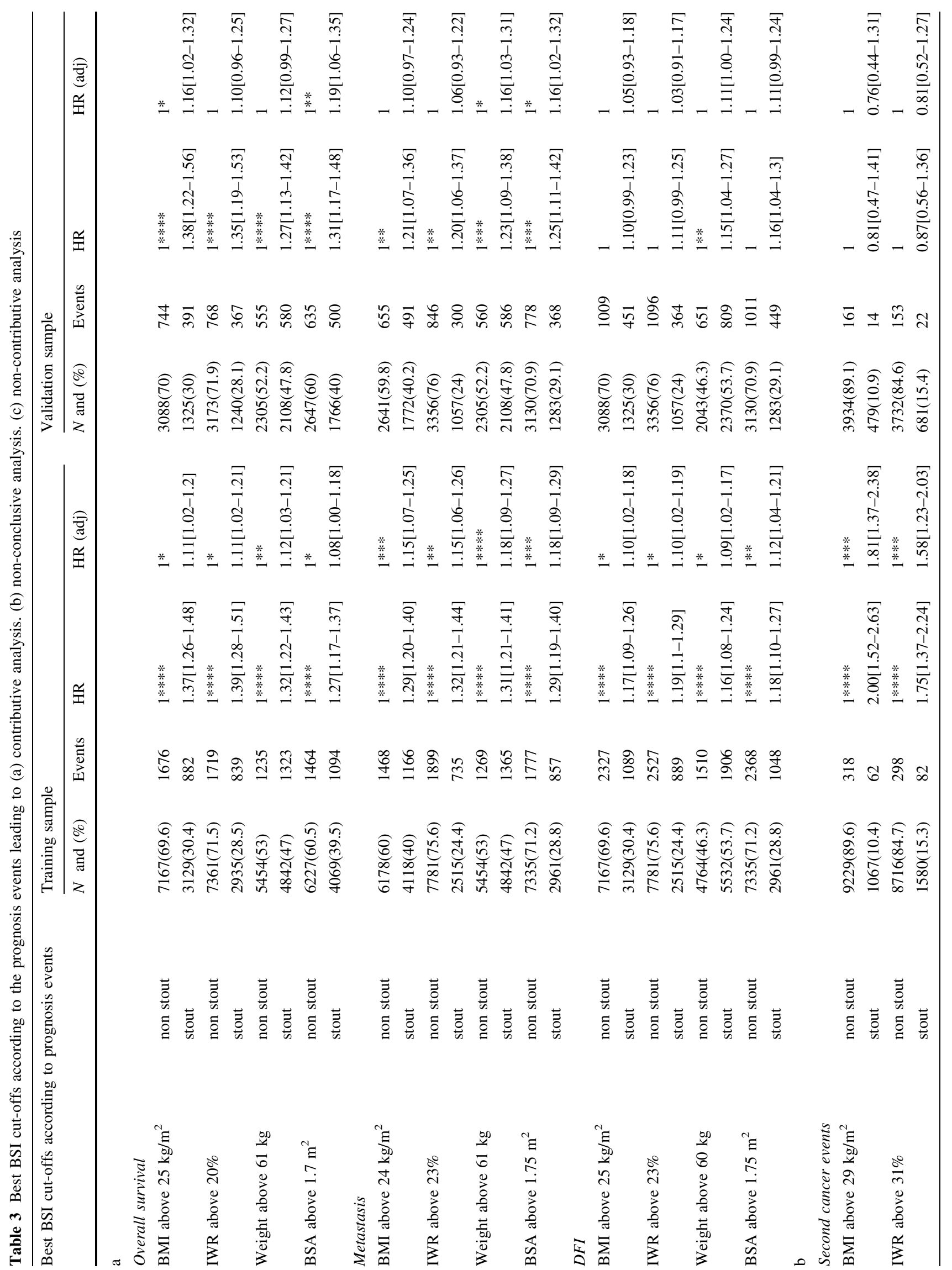




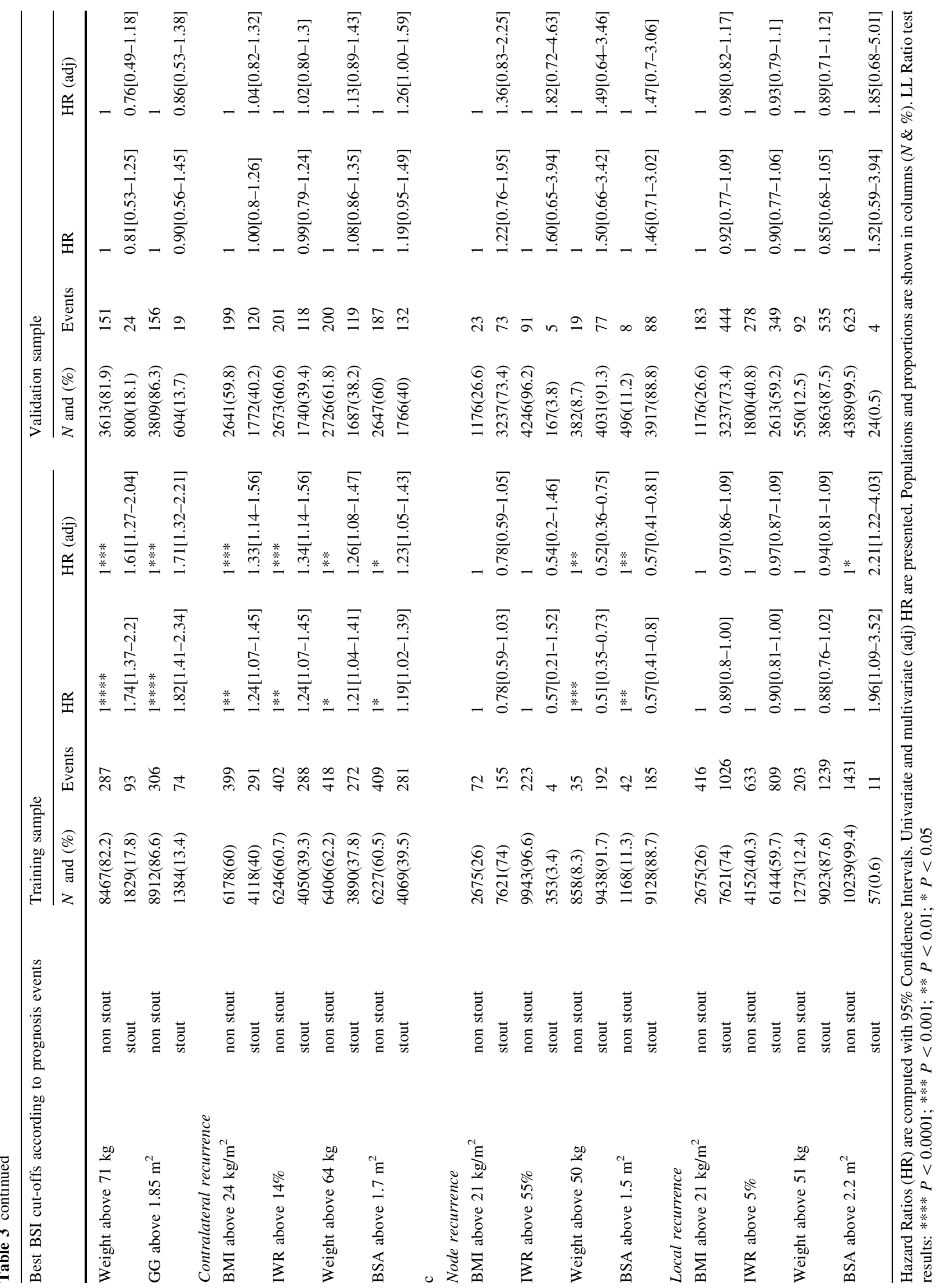


Table 4 Best body size indicators' cut-offs agreements according to the several prognosis events studied, kappa coefficients (top) and observed agreement (bottom)

\begin{tabular}{|c|c|c|c|c|c|c|c|c|c|}
\hline \multicolumn{5}{|c|}{ Overall survival events } & \multicolumn{5}{|c|}{ Metastasis recurrence } \\
\hline & $\operatorname{BMI}(25)$ & $\mathrm{W}(61)$ & $\operatorname{IWR}(20)$ & $\operatorname{BSA}(1.7)$ & & $\mathrm{BMI}(24)$ & $\mathrm{W}(61)$ & $\operatorname{IWR}(23)$ & $\operatorname{BSA}(1.75)$ \\
\hline $\operatorname{BMI}(25)$ & 1 & 0.59 & 0.9 & 0.58 & $\operatorname{BMI}(24)$ & 1 & 0.68 & 0.65 & 0.58 \\
\hline $\mathrm{W}(61)$ & 0.8 & 1 & 0.51 & 0.84 & $\mathrm{~W}(61)$ & 0.84 & 1 & 0.46 & 0.63 \\
\hline $\operatorname{IWR}(20)$ & 0.95 & 0.56 & 1 & 0.49 & $\operatorname{IWR}(23)$ & 0.84 & 0.74 & 1 & 0.54 \\
\hline $\operatorname{BSA}(1.7)$ & 0.81 & 0.92 & 0.64 & 1 & BSA(1.75) & 0.81 & 0.82 & 0.82 & 1 \\
\hline \multicolumn{5}{|c|}{ Second cancer events } & \multicolumn{5}{|c|}{ Contralateral recurrence } \\
\hline & BMI(29) & $\mathrm{W}(71)$ & $\operatorname{IWR}(31)$ & $\operatorname{BSA}(1.85)$ & & $\operatorname{BMI}(24)$ & $\mathrm{W}(64)$ & $\operatorname{IWR}(14)$ & $\operatorname{BSA}(1.7)$ \\
\hline $\operatorname{BMI}(29)$ & 1 & 0.63 & 0.48 & 0.6 & BMI(24) & 1 & 0.7 & 0.92 & 0.6 \\
\hline $\mathrm{W}(71)$ & 0.91 & 1 & 0.6 & 0.83 & $\mathrm{~W}(64)$ & 0.86 & 1 & 0.63 & 0.9 \\
\hline $\operatorname{IWR}(31)$ & 0.84 & 0.86 & 1 & 0.46 & $\operatorname{IWR}(14)$ & 0.96 & 0.82 & 1 & 0.53 \\
\hline $\operatorname{BSA}(1.85)$ & 0.91 & 0.95 & 0.82 & 1 & $\operatorname{BSA}(1.7)$ & 0.81 & 0.95 & 0.78 & 1 \\
\hline \multicolumn{5}{|c|}{ Nodes recurrence } & \multicolumn{5}{|c|}{ Local recurrence } \\
\hline & $\operatorname{BMI}(21)$ & $\mathrm{W}(50)$ & $\operatorname{IWR}(55)$ & $\operatorname{BSA}(1.5)$ & & $\operatorname{BMI}(21)$ & $\mathrm{W}(51)$ & $\operatorname{IWR}(5)$ & $\operatorname{BSA}(2.2)$ \\
\hline $\operatorname{BMI}(21)$ & 1 & 0.38 & 0.02 & 0.37 & $\mathrm{BMI}(21)$ & 1 & 0.49 & 0.68 & 0 \\
\hline $\mathrm{W}(50)$ & 0.81 & 1 & 0.01 & 0.8 & $\mathrm{~W}(51)$ & 0.84 & 1 & 0.3 & 0 \\
\hline $\operatorname{IWR}(55)$ & 0.29 & 0.12 & 1 & 0.01 & $\operatorname{IWR}(5)$ & 0.86 & 0.7 & 1 & 0.01 \\
\hline $\operatorname{BSA}(1.5)$ & 0.8 & 0.97 & 0.15 & 1 & $\operatorname{BSA}(2.2)$ & 0.27 & 0.13 & 0.41 & 1 \\
\hline
\end{tabular}

BMI: Body Mass Index in kg/m²; W: Weight in Kg; IWR: Ideal Weight Ratio; BSA: Body Surface Area according to the Gehan \& George formula in $\mathrm{m}^{2}$. BSI cut-off values are between parentheses

also effective as BMI's and kappa agreement coefficients between them are acceptable. These thresholds have already been used by several authors when taking WHO recommendations, studied populations BSI distributions or data from published literature into account. When compared to our findings, the results from other surveys are of a similar order of scale. For evaluations based on BMI, Galanis et al. and Daling et al. [1] used a threshold of $25.8 \mathrm{~kg} / \mathrm{m}^{2}$ to define their heaviest patients and to study overall survival. Kyogoku et al. [18] found that there was a $12 \%$ difference in survival likelihood between patients with a BMI of $20 \mathrm{~kg} / \mathrm{m}^{2}$ and those with a BMI of $24 \mathrm{~kg} / \mathrm{m}^{2}$, after a 10-year of follow-up. Kimura et al. [1] defined stout patients as those with a BMI of at least $23 \mathrm{~kg} / \mathrm{m}^{2}$, and revealed a significantly lower survival probability for stout post-menopausal women after a 10-year follow-up, when compared to leaner patients with a BMI lower than $21 \mathrm{~kg} / \mathrm{m}^{2}$. Using nonlinear modeling, Goodwin et al. [1] observed degraded disease free survival (DFS) and overall survival outcomes for patients with a BMI of at least $25 \mathrm{~kg} / \mathrm{m}^{2}$; nevertheless, these authors also observed poorer outcomes for patients with a BMI of less than $20 \mathrm{~kg} / \mathrm{m}^{2}$. In the modeling process of our population data, the use of a quadratic BMI term in addition to the linear term, did not suggest any increase of metastasis, DFI or overall survival events among leaner patients. With regard to the $\mathrm{W}$ cut-off values selected in our survey (60 and $61 \mathrm{~kg}$ ), various authors have used the same or very similar values to investigate overall breast cancer survival and DFS. Boyd et al. (64 kg cut-off), Donegan et al. (59 kg cut-off, unadjusted HR for DFS:1.4), Haybittle et al. (60 kg cut-off, unadjusted HR for OS: 1.7), Kyogoku et al. (60 kg cut-off, unadjusted HR for OS: 1.5 compared to patients under $45 \mathrm{~kg}$ ), Mohle-Boetani et al. (64 kg cut-off, adjusted HR for OS: 1.7 among pre-menopausal woman), Newman et al. (63 kg cut-off, unadjusted HR for OS: 1.7) have all observed significant associations between overweight and poorer breast cancer prognosis outcomes [1, 2, 19]. In the present study, we found HR values ranging between approximately 1.3 and 1.4 in our univariate analyses, which moved to the range 1.1-1.2 in multivariate analyses of overall survival and metastasis recurrence events. IWR is less frequently employed in published surveys; nonetheless, Bastarrachea et al. and Kyogoku et al. used a $20 \%$ IWR cut-off, whereas Senie et al. and Obermair et al. used a $25 \%$ IWR cut-off to investigate OS and DFS outcomes in breast cancer [1]. The estimated adjusted values for HR (about 1.3) given in the studies of Bastarrachea et al. and Senie et al. are very close to our results [1]. BSA is the least used BSI for the study of breast cancer prognosis; very few authors have reported a 
Table 5 Computed Log Likelihood, AIC and C-index enabling the selection of BSI cut-offs selection from unadjusted HR in the test sample

\begin{tabular}{|c|c|c|c|c|c|c|}
\hline \multirow{2}{*}{$\begin{array}{l}\text { BSI optimal cut-offs according } \\
\text { to prognosis events }\end{array}$} & \multicolumn{4}{|l|}{ Training sample } & \multicolumn{2}{|c|}{ Validation sample } \\
\hline & $\begin{array}{l}\text { Log-likelihood } \\
\text { (univariate) }\end{array}$ & $\begin{array}{l}\text { AIC } \\
\text { (univariate) }\end{array}$ & $\begin{array}{l}\text { C-index } \\
\text { (univariate) }\end{array}$ & $\begin{array}{l}\text { C-index } \\
\text { (multivariate) }\end{array}$ & $\begin{array}{l}\text { C-index } \\
\text { (univariate) }\end{array}$ & $\begin{array}{l}\text { C-index } \\
\text { (multivariate) }\end{array}$ \\
\hline \multicolumn{7}{|l|}{ Overall survival } \\
\hline BMI above $25 \mathrm{~kg} / \mathrm{m}^{2}$ & -22078.89 & 44159.78 & 0.534 & 0.748 & 0.535 & 0.740 \\
\hline IWR above $20 \%$ & -22076.76 & 44155.52 & 0.533 & 0.744 & 0.531 & 0.734 \\
\hline Weight above $61 \mathrm{~kg}$ & -22080.84 & 44163.69 & 0.534 & 0.749 & 0.529 & 0.743 \\
\hline BSA GG above $1.7 \mathrm{~m}^{2}$ & -22088.42 & 44178.84 & 0.524 & 0.748 & 0.531 & 0.743 \\
\hline \multicolumn{7}{|l|}{ Metastasis recurrence } \\
\hline BMI above $24 \mathrm{~kg} / \mathrm{m}^{2}$ & -23235.28 & 46472.57 & 0.533 & 0.743 & 0.520 & 0.741 \\
\hline IWR above $23 \%$ & -23237.05 & 46476.09 & 0.528 & 0.743 & 0.516 & 0.740 \\
\hline Weight above $61 \mathrm{~kg}$ & -23233.06 & 46468.12 & 0.535 & 0.744 & 0.526 & 0.742 \\
\hline BSA GG above $1.75 \mathrm{~m}^{2}$ & -23237.98 & 46477.96 & 0.521 & 0.744 & 0.526 & 0.739 \\
\hline \multicolumn{7}{|l|}{ Second cancer } \\
\hline BMI above $29 \mathrm{~kg} / \mathrm{m}^{2}$ & -3272.14 & 6546.27 & 0.542 & 0.596 & 0.506 & 0.550 \\
\hline IWR above $31 \%$ & -3273.74 & 6549.48 & 0.536 & 0.582 & 0.504 & 0.560 \\
\hline Weight above $71 \mathrm{~kg}$ & -3273.11 & 6548.22 & 0.545 & 0.571 & 0.500 & 0.538 \\
\hline BSA GG above $1.85 \mathrm{~m}^{2}$ & -3273.44 & 6548.89 & 0.541 & 0.575 & 0.505 & 0.536 \\
\hline \multicolumn{7}{|l|}{ Contralateral recurrence } \\
\hline BMI above $24 \mathrm{~kg} / \mathrm{m}^{2}$ & -5861.78 & 11725.56 & 0.526 & 0.558 & 0.512 & 0.532 \\
\hline IWR above $14 \%$ & -5861.83 & 11725.66 & 0.519 & 0.564 & 0.503 & 0.513 \\
\hline Weight above $64 \mathrm{~kg}$ & -5862.72 & 11727.43 & 0.517 & 0.554 & 0.509 & 0.535 \\
\hline BSA GG above $1.7 \mathrm{~m}^{2}$ & -5863.18 & 11728.37 & 0.513 & 0.556 & 0.524 & 0.532 \\
\hline \multicolumn{7}{|l|}{ Nodes recurrences } \\
\hline BMI above $21 \mathrm{~kg} / \mathrm{m}^{2}$ & -2019.93 & 4041.86 & 0.512 & 0.668 & 0.514 & 0.745 \\
\hline IWR above $55 \%$ & -2020.68 & 4043.35 & 0.503 & 0.672 & 0.501 & 0.748 \\
\hline Weight above $50 \mathrm{~kg}$ & -2015.62 & 4033.24 & 0.539 & 0.676 & 0.514 & 0.757 \\
\hline BSA GG above $1.5 \mathrm{~m}^{2}$ & -2016.66 & 4035.33 & 0.537 & 0.673 & 0.511 & 0.756 \\
\hline \multicolumn{7}{|l|}{ Local recurrence } \\
\hline BMI above $21 \mathrm{~kg} / \mathrm{m}^{2}$ & -12591.74 & 25185.48 & 0.511 & 0.689 & 0.507 & 0.688 \\
\hline IWR above $5 \%$ & -12591.80 & 25185.60 & 0.512 & 0.690 & 0.509 & 0.689 \\
\hline Weight above $51 \mathrm{~kg}$ & -12592.18 & 25186.37 & 0.502 & 0.685 & 0.505 & 0.696 \\
\hline BSA GG above $2.2 \mathrm{~m}^{2}$ & -12591.81 & 25185.62 & 0.503 & 0.691 & 0.500 & 0.695 \\
\hline \multicolumn{7}{|l|}{$D F I$} \\
\hline BMI above $25 \mathrm{~kg} / \mathrm{m}^{2}$ & -29919.09 & 59840.17 & 0.517 & 0.703 & 0.503 & 0.704 \\
\hline IWR above $23 \%$ & -29918.12 & 59838.23 & 0.517 & 0.702 & 0.508 & 0.709 \\
\hline Weight above $60 \mathrm{~kg}$ & -29918.72 & 59839.43 & 0.517 & 0.703 & 0.517 & 0.710 \\
\hline BSA GG above $1.75 \mathrm{~m}^{2}$ & -29918.07 & 59838.14 & 0.515 & 0.701 & 0.514 & 0.711 \\
\hline
\end{tabular}

The C-index from the adjusted HR in the test sample, and the C-index from the unadjusted and adjusted HR in the validation sample were also computed

significant relationship between BSA and breast cancer DFS [12] or OS [18]. In these cases, Gehan and George's formula [25] was employed and the highest used cut-point value was $1.5 \mathrm{~m}^{2}$, with an unadjusted HR of about 1.7 , when compared to patients with a BSA of less than $1.4 \mathrm{~m}^{2}$ [18].

Concerning second cancer events, the influence of significant overweight was estimated from our population training sample. The selected BMI threshold matches the obesity cut-point definition given by the WHO. Nonetheless, results from the validation sample did not confirm these findings. Dignam et al. [37] observed, from a substantial cohort of 3,385 patients, a significant increase in the occurrence of second cancers and contralateral breast cancers among women with a BMI above $30 \mathrm{~kg} / \mathrm{m}^{2}$, when compared to those with a BMI of less than $25 \mathrm{~kg} / \mathrm{m}^{2}$ : HR values of up to 1.60 were estimated. In the survey described in the present paper, when optimal BSI cut-offs were implemented, the 
estimated univariate HR for second primary cancers reached a value of 2, whereas adjusted HR values ranged between 1.6 and 1.8 , which is close to the HR range estimated given by Dignam et al. Among the patients in the validation sample defined as being stout, few events were noticed (about 20). Together with node recurrence, second primary cancer outcomes are the least observed events (Table 2). Although the $\mathrm{C}$-index values are clearly better, as far as the results from the training sample are concerned (thus supporting HR as an accurate indicator), HR discrepancies in the validation sample did not enable BSI cut-offs to be validated for second primary cancer outcomes. A similar situation was observed concerning the outcome of contralateral recurrences. Nevertheless, the use of the optimal $1.7 \mathrm{~m}^{2}$ BSA cut-point appears to be efficient, contrary to the use of other selected BSI cut-points are not; however, the former BSA threshold does not produce better outcome statistics (LL, AIC and C-Index). Suitable agreement between binary BSI corresponds to the WHO overweight cut-point definition. Although obesity is clearly recognized as a risk factor for breast cancer among post-menopausal women [38], these results do not permit BSI cut-points, derived from contralateral recurrence event analysis, to be validated.

Nodal and local recurrence events are rarely specifically studied. Marret et al. [1] found an independent prognostic role for BMI, when used as a continuous variable, by associating it with a reduced risk of local recurrence $(H R=0.92$ [0.85-0.99]). In the survey reported here, the investigation of local recurrence events led to a non-significant relationship between overweight and the risk of local recurrence. However, optimal BMI, W and IWR cutoffs enabled a reliable HR to be estimated, with a value a little less than unity (Table $3 \mathrm{c}$ ). When node recurrence events are considered, HR estimates from the validation sample are contradictory to those derived from the test sample. The IWR cut-point have very poor agreements with the other binary BSI, whereas W and BSA exhibit a "very good" agreement coefficient (0.8), better LL, AIC and $\mathrm{C}$-index values, and significant unadjusted and adjusted HR estimates from the training sample. However, adjusted HR estimates from the validation sample had clearly better $\mathrm{C}$-index values. Our interpretation of these contradictory results is that they are not consistent and are linked to an unconfirmed relationship between stoutness and breast cancer local or nodal recurrence. A simulation study has shown that the use of a cut-point, in presence of a continuous link between covariate and event risk, leads to a significant loss of power [34]. The continuous use of BSI in local or nodal recurrence risks was non-significant in our study. In the case of local and nodal recurrences, significant results appear to be related to sampling fluctuations, especially when the studied subpopulations have a small number of uncensored survival times [28].
The present study offers new data relevant to the use of optimal cut-points, for several different BSI. This is particularly accurate and contributive for OS, DFI and Metastasis recurrence, the most frequently observed events during breast cancer follow-up. Suggested optimal thresholds for second primary cancers and contralateral recurrence events were not validated, since the outcome of our analysis was not conclusive. While local and nodal recurrence event analyses were irrelevant, the influence of BSI on DFI events is related to the risk of metastasis recurrence. As shown in our results, even though various binary BSI can have equivalent efficiencies, their kappa agreement coefficients vary from "fair" to "very good" [36]. In our survey, this finding could not be explained by a kappa paradox [32]. Although optimally efficient binary BSI cut-points do not allow exactly the same patients to be identified, they are in substantial agreement. The results of our study can be used to improve the understanding of discrepancies between other studies, arising from the choice of BSI and cut-points.

The analysis methodology described in this study is based on usual statistical modeling tools for the selection of variables (log likelihood, AIC), to check the model's discriminatory ability (C-index) and to validate results (external validation by data splitting). The efficiency of this strategy has already been illustrated [39], and presents the advantage of simplified computations and interpretation of results, in the case of a substantially large population sample. Some authors have suggested selecting optimal cut-points by the use of cross validation procedures [34], bootstrapping computations of an optimal cut-point effect confidence interval [40, 41], adjusted significance level tests [34, 42] and multivariate settings [43]. However, loss of power [34, 43] and underestimated HR [34] have been reported in the case of the cross validation strategy. When only a limited population size is available, internal validation methods such as re-sampling techniques and bootstrapping [28] should obviously be preferred.

From our study, validated BSI cut-points from the investigation of overall survival and metastasis recurrence events support the use of a BMI value of $25 \mathrm{~kg} / \mathrm{m}^{2}$, a W value of $60 \mathrm{~kg}$, an IWR value of $20 \%$ and a BSA value of $1.7 \mathrm{~m}^{2}$ to identify stout patients with a poorer breast cancer prognosis. These cut-points may be used by health professionals, to conduct nutritional intervention studies for the improvement of patients' breast cancer prognosis.

Additional studies are needed to explore second primary cancer and contralateral recurrence events in order to validate optimal binary BSI cut-points. The improvement of breast cancer survival, over the last decades, should enable these required surveys. Further validation of optimal BSI cut-offs, in relation to OS and metastasis recurrence events among other populations [23], would also be useful. 


\section{References}

1. Rock CL, Demark-Wahnefried W (2002) Nutrition and survival after the diagnosis of breast cancer: a review of the evidence. J Clin Oncol 20(15):3302-3316. doi:10.1200/JCO.2002.03.008

2. Goodwin PJ, Boyd NF (1990) Body size and breast cancer prognosis: a critical review of the evidence. Breast Cancer Res Treat 16(3):205-214. doi:10.1007/BF01806329

3. Ryu SY, Kim CB, Nam CM, Park JK, Kim KS, Park J et al (2001) Is body mass index the prognostic factor in breast cancer? a meta-analysis. J Korean Med Sci 16(5):610-614

4. Carmichael AR, Bates T (2004) Obesity and breast cancer: a review of the literature. Breast 13(2):85-92. doi:10.1016/ j.breast.2003.03.001

5. Majed B, Moreau T, Senouci K, Salmon RJ, Fourquet A, Asselain B (2007) Is obesity an independent prognosis factor in woman breast cancer? Breast Cancer Res Treat

6. (2000) Obesity: preventing and managing the global epidemic. Report of a WHO consultation. World Health Organ Tech Rep Ser 894:i-xii, 1-253.

7. Gray DS, Fujioka K (1991) Use of relative weight and body mass index for the determination of adiposity. J Clin Epidemiol 44(6):545-550. doi:10.1016/0895-4356(91)90218-X

8. Garrow JS, Webster J (1985) Quetelet's index (W/H2) as a measure of fatness. Int J Obes 9(2):147-153

9. Frankenfield DC, Rowe WA, Cooney RN, Smith JS, Becker D (2001) Limits of body mass index to detect obesity and predict body composition. Nutrition 17(1):26-30. doi:10.1016/S08999007(00) 00471-8

10. Swinburn BA, Craig PL, Daniel R, Dent DP, Strauss BJ (1996) Body composition differences between Polynesians and Caucasians assessed by bioelectrical impedance. Int J Obes Relat Metab Disord 20(10):889-894

11. Stevens J, Juhaeri, Cai J, Jones DW (2002) The effect of decision rules on the choice of a body mass index cutoff for obesity: examples from African American and white women. Am J Clin Nutr 75(6):986-992

12. Boyd NF, Campbell JE, Germanson T, Thomson DB, Sutherland DJ, Meakin JW (1981) Body weight and prognosis in breast cancer. J Natl Cancer Inst 67(4):785-789

13. Borugian MJ, Sheps SB, Kim-Sing C, Olivotto IA, Van Patten C, Dunn BP et al (2003) Waist-to-hip ratio and breast cancer mortality. Am J Epidemiol 158(10):963-968. doi:10.1093/aje/kwg236

14. Donegan WL, Hartz AJ, Rimm AA (1978) The association of body weight with recurrent cancer of the breast. Cancer

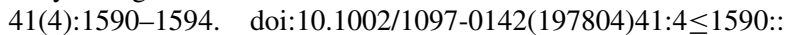
AID-CNCR2820410449 $\geq 3.0 . C O ; 2-\mathrm{N} \rightarrow>$

15. Enger SM, Greif JM, Polikoff J, Press M (2004) Body weight correlates with mortality in early-stage breast cancer. Arch Surg 139(9):954-958. doi:10.1001/archsurg.139.9.954 discussion 958-960

16. Goodwin PJ, Ennis M, Pritchard KI, Trudeau ME, Koo J, Madarnas Y et al (2002) Fasting insulin and outcome in early-stage breast cancer: results of a prospective cohort study. J Clin Oncol 20(1):42-51. doi:10.1200/JCO.20.1.42

17. Hebert JR, Augustine A, Barone J, Kabat GC, Kinne DW, Wynder EL (1988) Weight, height and body mass index in the prognosis of breast cancer: early results of a prospective study. Int J Cancer 42(3):315-318. doi:10.1002/ijc.2910420302

18. Kyogoku S, Hirohata T, Takeshita S, Nomura Y, Shigematsu T, Horie A (1990) Survival of breast-cancer patients and body size indicators. Int J Cancer 46(5):824-831. doi:10.1002/ijc.2910460513

19. Mohle-Boetani JC, Grosser S, Whittemore AS, Malec M, Kampert JB, Paffenbarger RS Jr (1988) Body size, reproductive factors, and breast cancer survival. Prev Med 17(5):634-642. doi: 10.1016/0091-7435(88) 90056-4
20. Bastarrachea J, Hortobagyi GN, Smith TL, Kau SW, Buzdar AU (1994) Obesity as an adverse prognostic factor for patients receiving adjuvant chemotherapy for breast cancer. Ann Intern Med 120(1):18-25

21. Senie RT, Rosen PP, Rhodes P, Lesser ML, Kinne DW (1992) Obesity at diagnosis of breast carcinoma influences duration of disease-free survival. Ann Intern Med 116(1):26-32

22. Suissa S, Pollak M, Spitzer WO, Margolese R (1989) Body size and breast cancer prognosis: a statistical explanation of the discrepancies. Cancer Res 49(11):3113-3116

23. Himes JH, Bouchard C, Pheley AM (1991) Lack of correspondence among measures identifying the obese. Am J Prev Med $7(2): 107-111$

24. Rock CL (2003) Diet and breast cancer: can dietary factors influence survival? J Mammary Gland Biol Neoplasia 8(1): 119-132. doi:10.1023/A:1025791523375

25. Gehan EA, George SL (1970) Estimation of human body surface area from height and weight. Cancer Chemother Rep 54(4):225-235

26. Therneau TM, Grambsch PM (2000) Modeling Survival Data: Extending the Cox Model, 1st edn. Springer-Verlag

27. Collett D (2003) Modelling survival data in medical research, 2nd edn. Chapman \& Hall/CRC, Boca Raton, Fla

28. Harrell FE (2001) Regression modeling strategies: with applications to linear models, logistic regression, and survival analysis. Springer, New York

29. Vittinghoff E (2005) Regression methods in biostatistics: linear, logistic, survival, and repeated measures models. Springer, New York

30. Harrell FE Jr, Califf RM, Pryor DB, Lee KL, Rosati RA (1982) Evaluating the yield of medical tests. JAMA 247(18):2543-2546. doi:10.1001/jama.247.18.2543

31. Pencina MJ, D'Agostino RB (2004) Overall C as a measure of discrimination in survival analysis: model specific population value and confidence interval estimation. Stat Med 23(13):21092123. doi:10.1002/sim. 1802

32. Viera AJ, Garrett JM (2005) Understanding interobserver agreement: the kappa statistic. Fam Med 37(5):360-363

33. Rosner B (2000) Fundamentals of biostatistics, 5th edn. Duxbury, Pacific Grove, CA

34. Faraggi D, Simon R (1996) A simulation study of cross-validation for selecting an optimal cutpoint in univariate survival analysis. Stat Med 15(20):2203-2213. doi:10.1002/(SICI)10970258(19961030)15:20 $\leq 2203:: A I D-S I M 357 \geq 3.0 . C O ; 2-G$

35. Porter GA, Inglis KM, Wood LA, Veugelers PJ (2006) Effect of obesity on presentation of breast cancer. Ann Surg Oncol 13(3):327-332. doi:10.1245/ASO.2006.03.049

36. Carpenter CR (2005) Kappa statistic. CMAJ 173(1):15-16. doi: 10.1503/cmaj.1041742 author reply 17

37. Dignam JJ, Wieand K, Johnson KA, Fisher B, Xu L, Mamounas EP (2003) Obesity, tamoxifen use, and outcomes in women with estrogen receptor-positive early-stage breast cancer. J Natl Cancer Inst 95(19):1467-1476

38. Renehan AG, Tyson M, Egger M, Heller RF, Zwahlen M (2008) Body-mass index and incidence of cancer: a systematic review and meta-analysis of prospective observational studies. Lancet 371(9612):569-578. doi:10.1016/S0140-6736(08) 60269-X

39. Hilsenbeck SG, Clark GM, McGuire WL (1992) Why do so many prognostic factors fail to pan out? Breast Cancer Res Treat 22(3):197-206. doi:10.1007/BF01840833

40. Abdolell M, LeBlanc M, Stephens D, Harrison RV (2002) Binary partitioning for continuous longitudinal data: categorizing a prognostic variable. Stat Med 21(22):3395-3409. doi:10.1002/ sim. 1266

41. Hollander N, Sauerbrei W, Schumacher M (2004) Confidence intervals for the effect of a prognostic factor after selection of an 'optimal' cutpoint. Stat Med 23(11):1701-1713. doi:10.1002/sim.1611 
42. Hollander N, Schumacher M (2001) On the problem of using 'optimal' cutpoints in the assessment of quantitative prognostic factors. Onkologie 24(2):194-199. doi:10.1159/000050315
43. Mazumdar M, Smith A, Bacik J (2003) Methods for categorizing a prognostic variable in a multivariable setting. Stat Med 22(4): 559-571. doi:10.1002/sim.1333 